\title{
iPS cells generated without c-Myc have active DIk1-Dio3 region and are capable of producing full-term mice through tetraploid complementation
}

\author{
Cell Research (2011) 21:550-553. doi:10.1038/cr.2011.25; published online 15 February 2011
}

\section{Dear editor,}

Due to its ethical acceptability and technical feasibility, production of induced pluripotent stem (iPS) cells from differentiated somatic cells by exogenous expression of several key transcription factors has given great promise to regenerative medicine and drug discovery [1]. The most commonly used transcription factors in iPS cell induction are Oct4, Sox2, Klf4 and c-Myc [2]. However, as $c-M y c$ is a proto-oncogene that causes many kinds of malignant tumors, whether it is safe to be used as an iPS cell induction factor has long been a concern. Previous studies have successfully produced iPS cells without using c-Myc, and obtained viable chimera mice with reduced tumorigenicity [3]. However, these threefactor-induced iPS cells had dramatically reduced induction efficiency and kinetics, and whether they could support full-term development of mice through tetraploid complementation remains unknown [4].

To further assess the feasibility of c-Myc free iPS cellinduction method and the pluripotency of resulted iPS cells, we infected mouse embryonic fibroblast (MEF) cells with three 'Yamanaka factors' excluding c-Myc [2], namely pMXs-Oct4, Sox2 and Klf4, using previously reported methods [5]. As the used MEF cells carried a transgenic Oct4-enhanced green florescence protein (GFP) reporter, whether they were reprogrammed back to the embryonic stem (ES) cell stage could be evaluated by examining the expression of the GFP protein (Figure 1A). At day 4 post infection, MEF cells were split on feeder cells and the culture was continued in a modified system, in which the knockout serum replacement (KOSR) was used to substitute the fetal bovine serum (FBS), as KOSR was shown to greatly improve both the efficiency and speed of reprogramming in our previous reports [6]. When examined at day 20 post infection, significantly higher ratio of GFP-positive cells was observed in the KOSR cell culturing system than in the FBS medium (Figure 1B, $17.49 \% \pm 5.56 \%$ vs $0.18 \% \pm 0.048 \%$ ).

Compared with the induction system using "Yamanaka factors" with c-Myc in previous reports $(24.04 \% \pm 7.6 \%$, ) [6], the three-factor method had a slightly lower reprogramming efficiency when cells were cultured in the same KOSR medium. The pace of reprogramming also slowed down in the three-factor system, in which the GFP-positive clones were first observed around day 14 post infection, whereas the same process only took 8 to 10 days in the four-factor system.

We picked large colonies with ES cell-like morphology and GFP expression to derive stable iPS cell lines (3F-iPS cells) at day 20 post infection (Figure 1A). Using primers specific for the virus-introduced exogenous transcription factor sequences (Supplementary information, Table S1), we performed PCR experiment and confirmed that there was no exogenous c-Myc integration in the 3F-iPS cell line IP20DT-3 (Figure 1C). A previously established iPS cell line with c-Myc and an ES cell line were included as controls. To characterize the pluripotent state of the 3F-iPS cells, we detected the expression of several ES cell marker genes using immunostaining assays. Pluripotent markers such as Oct4, Nanog and SSEA-1 were expressed as expected with correct subcellular locations (Figure 1D). Expression of other ES cell-specific genes, namely Rex1, Dppa3 and Gdf3, was also detected in the 3F-iPS cells, but not in the uninduced MEF cells (Supplementary information, Figure S1A). Further detection using primers specific for the amplification of endogenous or exogenous sequences of the 'Yamanaka factors' showed that the endogenous forms of these genes were activated, whereas the transgenes were silenced, indicating that the $3 \mathrm{~F}$-iPS cells were successfully reprogrammed to an ES cell-like stage (Supplementary information, Figure S1B).

To test whether the 3F-iPS cells could support the fullterm development of tetraploid-complemented mice, which was considered as the most rigorous test for cell pluripotency, we randomly selected two 3F-iPS cell lines to perform the tetraploid complementation assay. Viable full-term developed mice were obtained from one cell line 
A

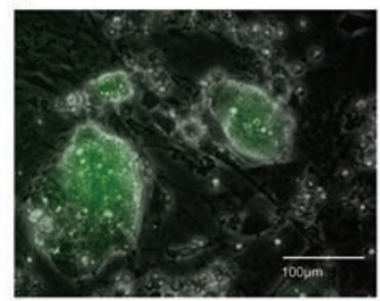

B

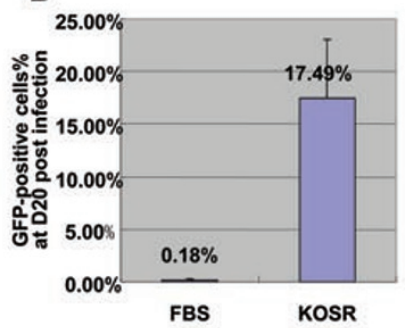

C

Tg-Oct4

Tg-Sox2

Tg-KII4

Tg-c-Myc

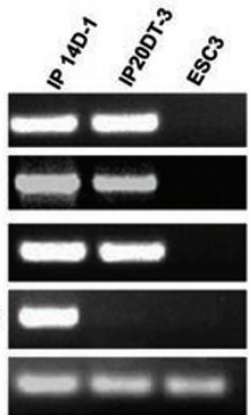

D

E
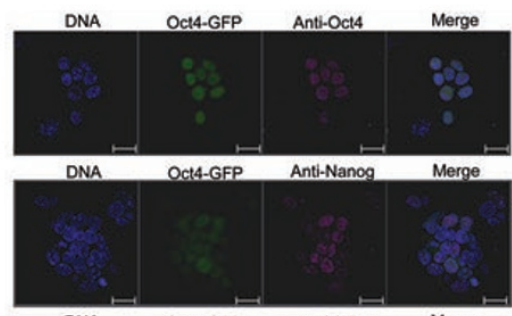

DNA OAt4-GEP AntissEA1 Merge
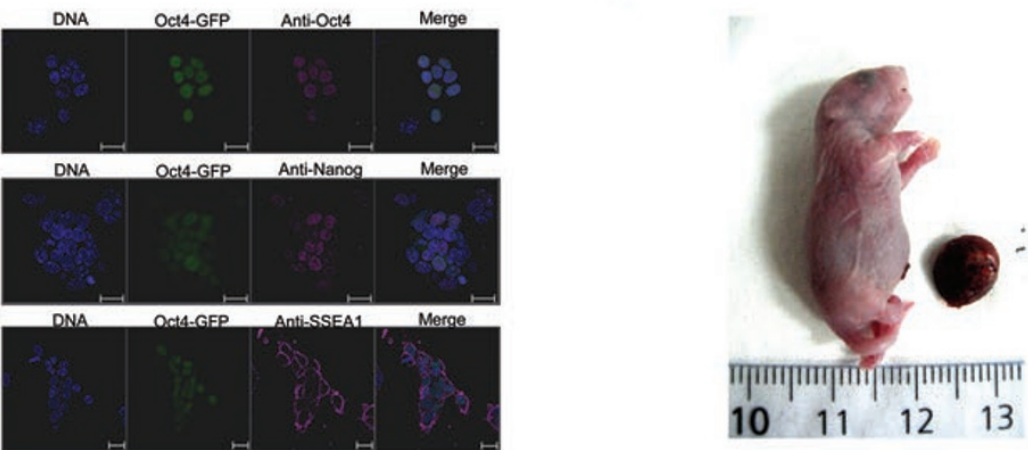

$\mathbf{F}$

Developmental efficiency of 3F-iPS cell line

\begin{tabular}{cccccc}
\hline cell line & $\begin{array}{c}\text { Injected } \\
\text { blastocysts }\end{array}$ & $\begin{array}{c}\text { Embryos arrested } \\
\text { before E10.5 }(\%)\end{array}$ & $\begin{array}{c}\text { Embryos arrested } \\
\text { at E10.5-E11.5 }(\%)\end{array}$ & $\begin{array}{c}\text { Embryos arrested } \\
\text { at E11.5-E13.5 }(\%)\end{array}$ & $\begin{array}{c}\text { Full term pups } \\
(\%)\end{array}$ \\
\hline IP20DT-3 & 318 & $203(63.8)$ & $7(2.2)$ & $4(1.26)$ & $4(1.26)$ \\
\hline IP20DT-4 & 179 & $113(63.2)$ & - & - & -
\end{tabular}

G

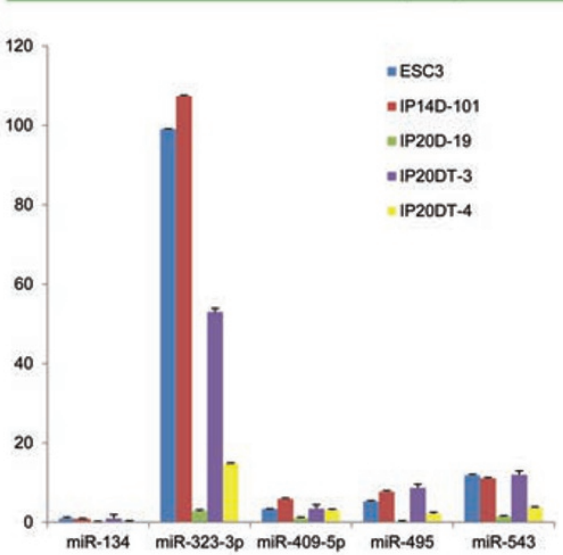

H

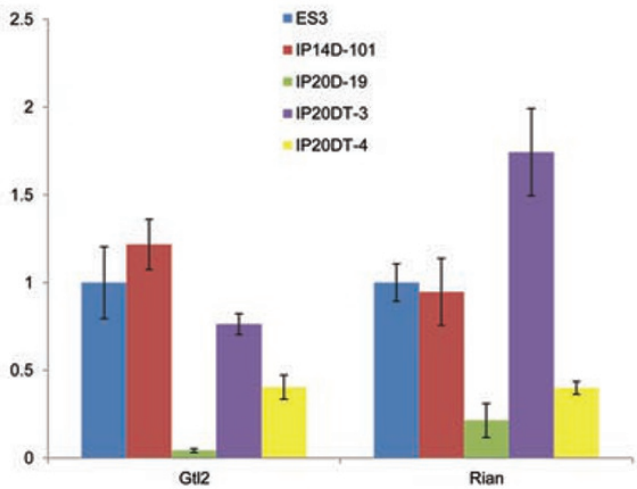

Figure 1 Generation and characterization of iPS cell lines without c-Myc. (A) 3F-iPS cell colonies with GFP expression driven by an Oct4 promoter. (B) Significant increase of GFP-positive cell ratio in KOSR medium compared with FBS medium at day 20 post infection of exogenous factors Oct4, Sox2 and Klf4, as detected by flow cytometry. (C) Genomic integration detection of exogenous sequences of the reprogramming factors showed no integration of transgene c-Myc. (D) Immunostaining of pluripotency markers Oct4, Nanog and SSEA-1 in 3F-iPS cell line IP20DT-3 showed correct gene expression locations. (E) Full-term mouse generated from 3F-iPS cell line IP20DT-3 through tetraploid complementation. (F) Developmental efficiency evaluation of two 3F-iPS cell lines through tetraploid complementation. (G) Quantitative real-time PCR detection of the expression of selected microRNAs located within the Dlk1-Dio3 region. (H) Quantitative real-time PCR detection of expression of two long non-coding RNA genes Gt/2 and Rian located within the DIk1-Dio3 region. ESC3, an ES cell line that passed tetraploid complementation assay; IP14D-101, a 4F-iPS cell line that passed tetraploid complementation assay; IP20D-19, a 4F-iPS cell line that failed to pass tetraploid complementation assay; IP20DT-3, a 3F-iPS cell line that passed tetraploid complementation assay; IP20DT-4, a 3F-iPS cell line that failed to pass tetraploid complementation assay. Detailed methods were provided as Supplementary information, Data S1. 
(Figure 1E and 1F). We previously demonstrated that the four 'Yamanaka factors' induced full pluripotent iPS cells could produce viable mice through tetraploid complementation with the same rate as that of the ES cells [7]. However, only $1.26 \%$ of $3 \mathrm{~F}-\mathrm{iPS}$ embryos of the cell line IP20DT-3 gave birth to full-term developed mice, as compared with the rate of $3.57 \%$ in the control ES cell embryos. No embryos developed beyond embryonic day 10.5 were obtained in the other 3F-iPS cell line, IP20DT-4, suggesting the lack of full pluripotency of this cell line (Figure $1 \mathrm{~F})$.

The activation of an imprinted genomic region, the Dlk1-Dio3 region, on mouse chromosome 12 has recently been demonstrated to be necessary for four factor induced iPS cells to obtain full pluripotency $[8,9]$. To test whether the activation of this region is also required for 3F-iPS cells to gain full pluripotency, we used realtime quantitative PCR to detect the expression of several microRNAs and other genes located within this region. Five microRNAs, namely miR-134, miR-323-3p, miR409-5p, miR-495 and miR-543, which were previously shown to be expressed in mouse ES and full pluripotent iPS cells (IP14D-101), but were not expressed in partially pluripotent iPS cells (IP20D-19), were all expressed in the 3F-iPS cell line IP20DT-3. The expression levels of these miRNAs in cell line IP20DT-3 was comparable with those observed in ES and four factor induced fullpluripotent iPS cells (Figure 1G). However, in the 3F-iPS cell line IP20DT-4, which could not support the development of full-term embryos, the expression of these miRNAs was much lower. Similar expression patterns were also observed for other two long non-coding RNAs encoded by this region, the Gtl2 and Rian genes (Figure $1 \mathrm{H})$. These results indicate that the pluripotency level of 3F-iPS cells also positively correlates with the activation of the Dlk1-Dio3 region.

The inclusion of oncogene c-Myc as one of the frequently used cell reprogramming factors has raised many safety concerns on the future clinical application of iPS cells. Although previous studies have shown that exogenous c-Myc was dispensable for iPS cell generation, the reprogramming progress became much slower and inefficient without exogenous c-Myc. More importantly, whether these $3 \mathrm{~F}-\mathrm{iPS}$ cells could support the full-term development of tetraploid-complemented mice remains a question. In this study, we successfully obtained 3F-iPS cells with high reprogramming efficiency by using the KOSR medium, and demonstrated that, similar to iPS cells induced with c-Myc, the 3F-iPS cells could also support the full-term development of tetraploid complemented mice. However, the full-term development rate of 3F-iPS embryos was much lower than that of ES cells and previously reported four factor induced full pluripotent iPS cells [6], indicating that most of the 3F-iPS cells may not be equivalent to ES cells. Our work also demonstrated that the activation of a previously reported marker region for the pluripotency of mouse iPS cells $[8$, 9], the Dlk1-Dio3 region, was also positively correlated with the pluripotency levels of 3F-iPS cells, indicating the feasibility of using this region as a marker to select full pluripotent 3F-iPS cells.

\section{Acknowledgments}

This study was supported in part by grants from the China National Basic Research Program (2006CB701501 to Q Z and 2007CB947702 to L W), and the National Science Foundation of China (90919060 to Q Z and 30725014 to X W).

Wei $\mathrm{Li}^{1,3, *}$, Xiao-yang Zhao ${ }^{1,3, *}$, Hai-feng Wan ${ }^{1,3}$, Ying Zhang ${ }^{1}$, Lei Liu ${ }^{1}$, Zhuo Lv ${ }^{1,3}$, Xiu-Jie Wang ${ }^{2}$, Liu Wang ${ }^{1}$, Qi Zhou ${ }^{1}$

${ }^{I}$ State Key Laboratory of Reproductive Biology, Institute of Zoology, Chinese Academy of Sciences, Beijing 100101, China; ${ }^{2}$ Center for Molecular Systems Biology, Institute of Genetics and Developmental Biology, Chinese Academy of Sciences, Beijing 100101, China $;{ }^{3}$ Graduate School of Chinese Academy of Sciences, Beijing 100049, China

*These two authors contributed equally to this work.

Correspondence: Qi Zhou ${ }^{\mathrm{a}}$, Liu Wang ${ }^{\mathrm{b}}$

${ }^{a}$ E-mail: qzhou@ioz.ac.cn

bE-mail: wangliu@ioz.ac.cn

\section{References}

1 Saha K, Jaenisch R. Technical challenges in using human induced pluripotent stem cells to model disease. Cell Stem Cell 2009; 5:584-595.

2 Takahashi K, Yamanaka S. Induction of pluripotent stem cells from mouse embryonic and adult fibroblast cultures by defined factors. Cell 2006; 126:663-676.

3 Nakagawa M, Koyanagi M, Tanabe K, et al. Generation of induced pluripotent stem cells without Myc from mouse and human fibroblasts. Nat Biotechnol 2008; 26:101-106.

4 Wernig M, Meissner A, Cassady JP, Jaenisch R. c-Myc is dispensable for direct reprogramming of mouse fibroblasts. Cell Stem Cell 2008; 2:10-12.

5 Zhao XY, Lv Z, Li W, Zeng F, Zhou Q. Production of mice using iPS cells and tetraploid complementation. Nat Protoc 2010; 5:963-971.

6 Zhao XY, Li W, Lv Z, et al. Efficient and rapid generation of induced pluripotent stem cells using an alternative culture medium. Cell Res 2010; 20:383-386.

7 Zhao XY, Li W, Lv Z, et al. iPS cells produce viable mice through tetraploid complementation. Nature 2009; 461:86-90.

8 Liu L, Luo GZ, Yang W, et al. Activation of the imprinted Dlk1-Dio3 region correlates with pluripotency levels of mouse 
stem cells. J Biol Chem 2010; 285:19483-19490.

9 Stadtfeld M, Apostolou E, Akutsu H, et al. Aberrant silencing of imprinted genes on chromosome $12 \mathrm{qF} 1$ in mouse induced pluripotent stem cells. Nature 2010; 465:175-181.

(Supplementary information is linked to the online version of the paper on the Cell Research website.) 